\title{
BMJ Open Use of behavioural activation to manage pain: a scoping review protocol
}

To cite: Walsh S, Jones M, Gray RJ, et al. Use of behavioural activation to manage pain: a scoping review protocol. BMJ Open 2021;11:e041036. doi:10.1136/ bmjopen-2020-041036

- Prepublication history and additional online supplemental material for this paper are available online. To view these files, please visit the journal online (http://dx.doi.org/10. 1136/bmjopen-2020-041036).

Received 10 June 2020 Accepted 06 May 2021
Check for updates

(C) Author(s) (or their employer(s)) 2021. Re-use permitted under CC BY-NC. No commercial re-use. See rights and permissions. Published by BMJ.

For numbered affiliations see end of article.

Correspondence to

Dr Martin Jones;

martin.jones@unisa.edu.au

\author{
Sandra Walsh (D), ${ }^{1}$ Martin Jones (D) , ${ }^{2}$ Richard John Gray (D) , ${ }^{3}$ \\ Marianne Gillam (D) ,2 Kate M Gunn, ${ }^{4}$ Trevor Barker, ${ }^{5}$ Tesfahun Eshetie, ${ }^{6}$ \\ G Lorimer Moseley ${ }^{7}$
}

\section{ABSTRACT}

Introduction Chronic pain is a distressing condition and often poorly treated and managed. Psychological therapies are considered first-line intervention for people with chronic pain. Common psychological therapies require extensive clinician training and specialist qualifications. One approach that does not need lengthy training nor specialist qualification, but has empirical support in other health domains, is behavioural activation (BA). BA seeks to increase engagement in behaviours that are valued by the person and progress through behaviours that can increase mood and develop skills that build satisfying routines. BA can help people to manage their condition through scheduling behaviours, promoting routine and mastery over their condition. The extent to which BA has been used to support people living with chronic pain is not clear. Methods and analysis This scoping review aims to identify published studies describing the application of BA to support people living with chronic pain. To map the evidence regarding BA and chronic pain, including the study type and the associated evidence, a scoping review was adopted. The search will be conducted in bibliographic databases, clinical trial registries and grey literature. No date limits will be applied to the search strategy. Screening of titles and abstracts, and full-text screening, will be independently undertaken by two investigators using Covidence software. Any disagreement between investigators will be resolved by a third investigator. Data from included publications will be extracted using a customised data extraction tool.

Ethics and dissemination The scoping review is an analysis of existing data and therefore ethics approval is not required. The findings of this scoping review will further our understanding of how BA has been used to support people living with chronic pain and inform future training and education programmes in this area.

\section{INTRODUCTION}

Globally, over one-third of the population will experience chronic pain in their lifetime. ${ }^{1}$ It impacts people's quality of life, overall functioning and mental health. Chronic pain shares a complex relationship with mental health issues, including depression and anxiety. In one of the largest population studies of pain and depression and anxiety, a significant association was found between chronic pain conditions

\section{Strengths and limitations of this study}

- This is the first scoping review that provides a comprehensive summary of published literature on the application of behavioural activation (BA) as an approach for chronic pain.

- The proposed review will provide insight on how BA could be used with people living with chronic pain and assist in the development of a BA protocol that can be tested for feasibility, safety and effectiveness.

- Only studies that focus on BA for adults living with chronic pain will be included.

- Excluding studies in in-patient hospital settings and paediatric chronic pain means that the results of the scoping review may not be applicable to these groups of population.

and depression-anxiety disorders status. ${ }^{1}$ However, they note that most $(80 \%)$ people living with a chronic pain condition did not meet the criteria for either a depressive or anxiety disorder. Broadly speaking, there is evidence that suggests that depression can lead to chronic pain, while chronic pain can lead to depression. ${ }^{2}$ The complexity of the relationship can make it challenging to characterise and conceptualise. ${ }^{3}$ Equally, the extent of this relationship has been difficult to determine. Mazzucchelli and Da ilva suggest that estimates of the association range from $40 \%$ to $60 \%{ }^{2}$

The inter-relationship with anxiety is less understood and often overlooked in research; however, there is emerging research that suggests this is worthy of attention. ${ }^{1}$ The connection between mood disorders and anxiety is well established, what is less known is how this interacts with pain. For posttraumatic stress disorder, the linkage is clearer when the source of both pain and psychological disturbance is related to an event causing physical issues, such as car accident. However, studies examining irritable bowel syndrome (IBS) have found that anxiety is a significant risk factor for IBS, ${ }^{4}$ and in follow-up care, approximately two-thirds of patients with IBS 
have symptoms of psychological distress, predominantly anxiety. ${ }^{5}$

The complexity of these conditions often requires a combination of psychological and pharmacological approaches. Psychological therapies are considered firstline intervention for chronic pain ${ }^{6}$ and the most widely studied, and implemented, psychological approaches are cognitive-behavioural therapies $(\mathrm{CBT}){ }^{7}$ In a recent metaanalysis of physical and CBT intervention programmes for chronic musculoskeletal pain, the authors noted inconsistent effects of exercise combined with CBT interventions on pain intensity; although there was evidence for this combined intervention minimising the impact of pain on everyday life. ${ }^{8}$ Interestingly, they found 'little evidence that interventions guided by CBT are better than physical exercise alone in improving mood' (p. 26). Other meta-analyses have compared CBT with education ${ }^{9}$ and acceptance-based interventions ${ }^{10}$ finding none superior to the other.

In a meta-analysis of 23 randomised controlled trials (RCTs) involving 3359 participants with lower back pain, compared CBT approaches to waitlist/usual care or other guideline-based active treatments, such as exercise and the provision of information. ${ }^{11}$ Study outcomes included pain, disability, quality of life, or work disability. At long term follow-up, examining pain as the outcome, the authors identified a small effect size (standardised mean difference (SMD) 0.23 CI 95\%) in favour of CBT when compared with waitlist / usual care. While the guidelinebased treatment favoured CBT as treatment, with a moderate effect size (SMD 0.48CI 95\%). ${ }^{11}$

The successful delivery of currently popular psychological therapies requires extensive training ${ }^{12}$ and 'takes a skillful therapist to be effective. A competent cognitive behaviour therapist will have had substantial training and experience in the area'. ${ }^{13}$ Globally, this is a substantial barrier - such highly trained and experienced health professionals are commonly not available in rural and remote areas and are expensive. For example, in major Australian cities, there are 120 psychologists for every 100000 people, but in rural and remote areas, this ratio drops to 12 psychologists for every 100000 people. ${ }^{14}$

There is clearly an imperative to develop and test psychological therapies that can be implemented widely and at limited cost. One potential therapy is Behavioural Activation (BA). BA draws on aspects of CBT; it aims to increase engagement in valued activities and decrease periods of inactivity; it involves monitoring mood and activity, identifying relationships between them, and scheduling more of those activities that improve mood. It has been hypothesised that depression is associated with reduced environmental reinforcers. This can be explained by people who are depressed engage less frequently in pleasant activities which result in positive changes in the person's environment. ${ }^{15} \mathrm{BA}$ is an effective treatment for depression. Its effect is explained by activation (activity) and the associated environment award which occurs with a change in increased activity. ${ }^{16}$ Kanter has argued that activity is informed by the person's values and the importance of incorporating the person's values into care and treatment plans. ${ }^{16}$ While Mazzucchelli and Da Silva ${ }^{2}$ offered that if pain is considered within operant modelling, as a treatment, BA is a 'good theoretical match' (p.5).

A systematic review examined mediators that could explain the effectiveness of BA. ${ }^{17}$ Fourteen studies were included: ten RCTs involving 1252 patients and four uncontrolled studies involving 94 patients. The mediators described were activity, using the BA for Depression Scale, and environmental influences, measured using the Environmental Reward Observation Scale. However, the quality of the evidence reported made firm conclusions. Highlighting the mediators explaining its efficacy for depression and other conditions, such as pain, warrants further investigation.

A systematic review and meta-analysis of 26 RCTs involving 1524 people with depression symptoms reported that $\mathrm{BA}$ was superior to control interventions with a large effect size $(\mathrm{SMD}=-0.74,95 \% \mathrm{CI}-0.91$ to -0.56$) .{ }^{18}$ In the same review, the effectiveness of BA against antidepressant medication was tested. Four trials involving 283 participants tested BA against antidepressant medication. The SMD at post-treatment was -0.42 , a moderate effect size in favour of BA. ${ }^{18}$

$\mathrm{BA}$ is as equally effective as $\mathrm{CBT}$ in treating depression. In a randomised controlled non-inferiority trial, Richards et al compared CBT, delivered by a highly trained clinicians with postgraduate qualifications in CBT, with a comparable dose of BA delivered by junior mental health workers with no formal qualification in psychology. ${ }^{19}$ Participants included 440 people with moderate to severe depression were randomly allocated to treatment. The treatments were equally effective in treating depression symptoms. Importantly, those who provided BA therapy received 5 days of training in $\mathrm{BA}$ and reached competency standards in delivering the intervention, before the trial commenced. ${ }^{19}$

The only psychological therapies recommended in the draft National Institute for Health and Care Excellence guidelines for the treatment of chronic pain are CBT and acceptance and commitment Therapy. ${ }^{20}$ As BA has been shown to be comparable to CBT in the treatment of depression, we are considering whether BA may be as beneficial as CBT in the treatment of chronic pain. BA may provide an effective option for the many millions of people living with chronic pain who are currently unable to access specialists for CBT. Although the mechanism through which this occurs could be debatable. For example, it could reduce depression and as a result reduce perceived pain severity; or, through operant conditioning, it could pair a sense of mastery/achievement with an activity that may have been pair with pain. The aim of this scoping review is to identify published studies describing the application of BA to adults living with chronic pain. To map the evidence generated in relation to BA and chronic pain, including the study types and the evidence available, a scoping review methodology 
was selected. Scoping reviews are considered appropriate if the intent is to scope the body of literature, explore the research conducted and refine/clarify concepts. ${ }^{21}$ The review will encompass studies that may have used BA to increase activity, quality of life and/or coping; or reduce pain intensity, pain-related disability, pain anxiety, depression and/or anxiety.

\section{METHODS AND ANALYSIS \\ Design}

This scoping review will use the methodological approach proposed by Peters $e t a l,{ }^{22}$ which extends the Arksey and $\mathrm{O}^{\prime}$ Malley ${ }^{23}$ framework. The approach will consist of the following stages: identifying review questions, determining the selection criteria and search strategy, undertaking extraction, charting, appraisal and reporting of the results. Each stage is further described below.

\section{Identifying review questions}

The review aims to identify studies that use BA to support patients living with chronic pain. Specifically, the review questions are:

1. What studies have been published on the use of BA to support patients living with chronic pain?

2. How has BA been applied to support patients with chronic pain?

3. How has BA been integrated with other models of care/treatments for people living with chronic pain?

\section{Determining selection criteria}

Population

We will include studies with patients over the age of 18 with chronic pain. Young people with chronic pain will be excluded as the management of pain in this population can be different to the adult population. The cognitive development of young people may influence the application of different psychological therapies.

\section{Concept}

We want to understand how BA has been used to support people living with chronic pain conditions. We want to understand how BA has been used to support people living with chronic pain conditions. Given that chronic pain covers an array of conditions and disorders, we are interested in any subpopulations (such as people with back pain, musculoskeletal disorders/conditions, cancer). We are interested in all studies that examine the use of BA with this population and anticipate these studies may focus on BA as a means to increase activity, quality of life and/or coping; or reduce pain intensity, pain-related disability, pain anxiety, depression and/or anxiety. We will also consider the nature of the intervention, including the mode and length of delivery.

Context

Our context is people with chronic pain who live in their own home or a residential care facility. These care settings are candidate settings for healthcare professionals to deliver BA and may be able to guide healthcare professionals on how best to deliver BA. We will exclude studies that include the delivery of BA for people with chronic pain in hospital settings. In-patient hospital settings will be excluded as presentations are typically for acute issues and psychological therapies, such as BA, are less likely to occur in these settings.

\section{Types of studies}

We will include the literature based on our inclusion criteria. We will include observational studies, experimental studies, case studies, clinical audits, systematic reviews, and opinion papers (which include guidelines in the use of BA in the management of pain).

\section{Search strategy}

The search will be conducted using an iterative process, with the assistance of a librarian. This will include the following three steps:

Step 1: As recommended by Arksey and O'Malley, ${ }^{23}$ we have searched one online database relevant to applying healthcare intervention. The Medline search strategy is detailed in online supplemental appendix 1 . We will use keywords, Medical Subject Headings (MeSH and index terms to help us design our next step.

Step 2: We will then use the identified keywords to develop the strategy across all the included databases.

Step 3: The search strategy will be developed for each database, and only include studies in English. The search will have no date limits applied. We will search the following databases: MEDLINE, Ovid EMCARE, CINAHL, PsycINFO, Cochrane Central Register of Controlled Trials, Scopus and Web of Science. The full search will be completed by 1 June 2021 .

\section{Study selection}

We will transfer and upload all citations to Endnote (Clarivate Analytics, Boston, Massachusetts, USA), and remove duplicates. We will import these studies into Covidence ( www.covidence.org). Title and abstract will be screened by two investigators against the inclusion criteria for the review. We have allowed 10 days for title and abstract screening. Full-text screening will follow a similar dual investigator process. Disagreements that arise between the reviewers will be resolved by a third investigator. If studies are excluded at full text screening, the reason/s will be noted. Full-text screening will be completed by 18 June 2021. The reporting of the scoping review will be informed by Preferred Reporting Items for Systematic Reviews and Meta-Analyses extension for Scoping Reviews Checklist (PRISMA-ScR) ${ }^{24}$ The proposed structure for the final scoping review is detailed on the PRISMA-ScR checklist included in online supplemental appendix 2. The results of the search will be reported in full in the final report and presented in a PRISMA flow diagram.

\section{Data extraction}

We will report details about the interventions, populations, study methods and outcomes of significance to the 
review question and review objectives. We will write to authors to request missing or additional data as required. We have drafted a charting table (data extraction form) (online supplemental appendix 3) to record information of the source, such as author, reference and results or findings relevant to the review question/s. We have tested the extraction form on three studies from the initial search to ensure all relevant results were extracted. We will make iterative improvements to the charting of results in this scoping review as required. Data extraction will be completed by 30 June 2021 .

\section{Data charting and synthesis}

We will map in narrative/tabular format/diagrammatic format to the review questions using the extraction chart (online supplemental appendix 3). This will provide a transparent summary of the studies and provide clear answers to the specific subquestions (and thereby the overarching research question) of this scoping review. It is anticipated that the final paper will be completed by 31 July 2021.

\section{Critical appraisal of sources of evidence}

The following tools will be used to critically appraise the studies located.

\begin{tabular}{ll}
\hline Type of study & Critical appraisal tool \\
\hline $\begin{array}{l}\text { Randomised } \\
\text { controlled trials }\end{array}$ & Risk of Bias $2^{25}$ \\
$\begin{array}{l}\text { Non-randomised } \\
\text { experimental studies }\end{array}$ & $\begin{array}{l}\text { JBI Critical Appraisal Checklist for } \\
\text { Quasi-Experimental Studies }\end{array}$ \\
Pre-poststudies & $\begin{array}{l}\text { National Institutes of Health quality } \\
\text { assessment tool for before-after (pre- } \\
\text { post) study with no control group }\end{array}$ \\
Case studies & Checklist for Case Reports ${ }^{28}$ \\
\hline
\end{tabular}

\section{Patient and public involvement}

Two people with a lived experience of chronic pain were involved in protocol and question development, relevance to the lived experience, appropriateness of language potential contribution to the field. Both were invited to stay involved in this project throughout.

\section{DISCUSSION}

Chronic pain is a public health concern requiring effective interventions. According to years lived with disability, it is the most burdensome health condition we face. ${ }^{29}$ In high-income countries such as Australia, in terms of years lost due to ill health, chronic pain is second only to ischaemic heart disease. ${ }^{29}$ There is a pressing need to develop, test and implement effective interventions that can reach a large proportion of the population despite geographical and economic accessibility limitations. This scoping review will help us to understand the potential of $\mathrm{BA}$ as a candidate intervention for people living with chronic pain. We will use the evidence from the scoping review to develop a BA training programme and cocreate a development and evaluation framework with consumers, carers, potential BA deliverers and healthcare professionals.

\section{Strengths and limitations}

This is the first scoping review that will offer a comprehensive summary of the published literature on the application of BA as an approach for chronic pain. The proposed review will provide insight on how BA could be used with people living with chronic pain and assist in the development of a BA protocol that can be tested for feasibility, safety, effectiveness and economic impact. This scoping review will only examine studies that focus on BA for adults living with chronic pain. This means that results may not be applicable to paediatric populations with chronic pain. Further, studies conducted in in-patient hospital settings will be excluded so the findings of this scoping review may not be relevant to people in this setting.

\section{ETHICS AND DISSEMINATION}

As a scoping review of published scientific literature, no ethical approval is required. The results of this review will be published in a peer-reviewed journal and presented at conferences relevant to pain management.

\section{Author affiliations}

${ }^{1}$ Department of Rural Health, University of South Australian - Whyalla Campus, Whyalla Norrie, South Australia, Australia

${ }^{2}$ Department of Rural Health, University of South Australia, Mt Barker, South Australia, Australia

${ }^{3}$ School of Nursing, La Trobe University, Bundoora, Victoria, Australia

${ }^{4}$ Cancer Research Institute, University of South Australia, Adelaide, South Australia, Australia

${ }^{5}$ Allied Health \& Human Performance, University of South Australia Division of Health Sciences, Adelaide, South Australia, Australia

${ }^{6}$ Department of Rural Health, University of South Australia Division of Health Sciences, Adelaide, South Australia, Australia

${ }^{7}$ Allied Health \& Human Performance, University of South Australia, Adelaide, South Australia, Australia

\section{Twitter Richard John Gray @drrichardgray}

Contributors MJ, SW, RJG, MG, KMG, TB, TE and GLM conceptualised the project, drafted the manuscript, contributed to the development of the research questions, study design and reviewed and edited the manuscript for important intellectual content. MJ, SW, RG, MG, KMG, TB, TE and GLM authors approved the final manuscript and accepted to be accountable for all aspects of the work.

Funding This study was supported by infrastructure provided by the Department of Rural Health, University of South Australia. GLM is supported by a Leadership Investigator Grant from the National Health \& Medical Research Council of Australia ID1178444.

Competing interests GLM has received support from: ConnectHealth UK, Seqirus, Kaiser Permanente, Workers' Compensation Boards in Australia, Europe and North America, AIA Australia, the International Olympic Committee, Port Adelaide Football Club, Arsenal Football Club. Professional and scientific bodies have reimbursed him for travel costs related to presentation of research on pain at scientific conferences and symposia. He has received speaker fees for lectures on pain and rehabilitation. He receives book royalties from NOlgroup publications, Dancing Giraffe Press \& OPTP, for books on pain science, education and rehabilitation.

Patient consent for publication Not required.

Provenance and peer review Not commissioned; externally peer reviewed. 
Supplemental material This content has been supplied by the author(s). It has not been vetted by BMJ Publishing Group Limited (BMJ) and may not have been peer-reviewed. Any opinions or recommendations discussed are solely those of the author(s) and are not endorsed by BMJ. BMJ disclaims all liability and responsibility arising from any reliance placed on the content. Where the content includes any translated material, BMJ does not warrant the accuracy and reliability of the translations (including but not limited to local regulations, clinical guidelines, terminology, drug names and drug dosages), and is not responsible for any error and/or omissions arising from translation and adaptation or otherwise.

Open access This is an open access article distributed in accordance with the Creative Commons Attribution Non Commercial (CC BY-NC 4.0) license, which permits others to distribute, remix, adapt, build upon this work non-commercially, and license their derivative works on different terms, provided the original work is properly cited, appropriate credit is given, any changes made indicated, and the use is non-commercial. See: http://creativecommons.org/licenses/by-nc/4.0/.

\section{ORCID iDs}

Sandra Walsh http://orcid.org/0000-0001-9702-652X

Martin Jones http://orcid.org/0000-0002-6463-3574

Richard John Gray http://orcid.org/0000-0001-9694-4206

Marianne Gillam http://orcid.org/0000-0003-0169-3964

\section{REFERENCES}

1 Tsang A, Von Korff M, Lee S, et al. Common chronic pain conditions in developed and developing countries: gender and age differences and comorbidity with depression-anxiety disorders. J Pain 2008;9:883-91.

2 Mazzucchelli TG, Da silva M. The potential of behavioural activation for the treatment of chronic pain: an exploratory review. Clin Psychol 2016;20:5-16.

3 Nicolson SE, Caplan JP, Williams DE, et al. Comorbid pain, depression, and anxiety: multifaceted pathology allows for multifaceted treatment. Harv Rev Psychiatry 2009;17:407-20.

4 Surdea-Blaga T, Băban A, Dumitrascu DL. Psychosocial determinants of irritable bowel syndrome. World J Gastroenterol 2012;18:616-26.

5 Tang Q-L, Lin G-Y, Zhang M-Q. Cognitive-Behavioral therapy for the management of irritable bowel syndrome. World J Gastroenterol 2013;19:8605-10.

6 Oliveira CB, Maher CG, Pinto RZ, et al. Clinical practice guidelines for the management of non-specific low back pain in primary care: an updated overview. Eur Spine J 2018;27:2791-803.

7 Turk DC, Meichenbaum D, Genest M. Pain and behavioural medicine: a cognitive-behavioral perspective. New York, NY: Guilford Press, 1983.

8 Cheng JOS, Cheng S-T. Effectiveness of physical and cognitivebehavioural intervention programmes for chronic musculoskeletal pain in adults: a systematic review and meta-analysis of randomised controlled trials. PLoS One 2019;14:E0223367.

9 Makhlouf S, Silva A, Khan S. Cognitive behavioural therapy versus education of adult patients with chronic pain. A meta-analysis of the change of pain intensity post-intervention and after 6 months. British Pain Society ASM.

10 Veehof MM, Oskam M-J, Schreurs KMG, et al. Acceptance-based interventions for the treatment of chronic pain: a systematic review and meta-analysis. Pain 2011;152:533-42.
11 Richmond H, Hall AM, Copsey B, et al. The effectiveness of cognitive behavioural treatment for non-specific low back pain: a systematic review and meta-analysis. PLoS One 2015;10:e0134192.

12 Dobmeyer AC. Psychological treatment of medical patients in integrated primary care. Washington, DC: American Psychological Association, 2018.

13 Australian Association for Cognitive Behaviour Therapy. What is CBT? [Internet], 2020. Available: https://www.aacbt.org.au/what-iscbt/ [Accessed May 2020].

14 Health Workforce Australia. Australia's Health Workforce Series Psychologists in Focus (2015). Canberra, Aust: Health Workforce Australia, 2015.

15 Lewinsohn PM. A behavioral approach to depression. essential papers on depression. New York: New York University Press, 1974: 150-72.

16 Kanter JW, Puspitasari AJ. Global dissemination and implementation of behavioural activation. Lancet 2016;388:843-4.

17 Janssen NP, Hendriks G-J, Baranelli CT. How does behavioural activation work? A systematic review of the evidence on potential mediators. Psychother Psychosom 2021;90:1-9.

18 Ekers D, Webster L, Van Straten A, et al. Behavioural activation for depression; an update of meta-analysis of effectiveness and sub group analysis. PLoS One 2014;9:e100100.

19 Richards DA, Ekers D, McMillan D, et al. Cost and outcome of behavioural activation versus cognitive behavioural therapy for depression (cobra): a randomised, controlled, non-inferiority trial. Lancet 2016;388:871-80.

20 National Institute for Health and Care Excellence. Chronic pain: assessment and management, 2020. Available: https://www.nice.org. uk/guidance/ng193/documents/evidence-review-6

21 Munn Z, Peters MDJ, Stern C, et al. Systematic review or scoping review? guidance for authors when choosing between a systematic or scoping review approach. BMC Med Res Methodol 2018;18:143.

22 Peters MDJ, Godfrey CM, Khalil H, et al. Guidance for conducting systematic scoping reviews. Int J Evid Based Healthc 2015;13:141-6.

23 Arksey H, O'Malley L. Scoping studies: towards a methodological framework. Int J Soc Res Methodol 2005;8:19-32.

24 Tricco AC, Lillie E, Zarin W, et al. PRISMA extension for scoping reviews (PRISMA-ScR): checklist and explanation. Ann Intern Med 2018;169:467-73.

25 Cochrane Methods. Risk of bias 2 (rob 2) tool, 2021. Available: https://methods.cochrane.org/risk-bias-2

26 Joanna Briggs Institute. The Joanna Briggs Institute critical appraisa tools for use in JBI systematic reviews: critical appraisal checklist for quasi-experimental studies, 2017. Available: https://jbi.global/sites/ default/files/2019-05/JBI_Quasi-Experimental_Appraisal_Tool2017_0. pdf

27 National Institutes of Health (NIH). National Institutes of health quality assessment tool for before-after (pre-post) study with no control group, 2020. Available: https://www.nhlbi.nih.gov/health-topics/ study-quality-assessment-tools

28 Joanna Briggs Institute. The Joanna Briggs Institute critical appraisal tools for use in JBI systematic reviews: checklist for case reports. 2017. The Joanna Briggs Institute critical appraisal tools for use in JBI systematic reviews.

29 James, SL, Abate, D, Abate, KH, et al. Global, regional, and national incidence, prevalence, and years lived with disability for 354 diseases and injuries for 195 countries and territories, 1990-2017: a systematic analysis for the global burden of disease study 2017. Lancet 2018;392:1789-858 https://www.thelancet.com/action/ showPdf?pii=S0140-6736\%2818\%2932335-3 